\title{
Mining Patient Flow Patterns in a Surgical Ward
}

\author{
Christoffer O. Back ${ }^{1} \mathbb{1}^{\mathrm{a}}$, Areti Manataki ${ }^{2} \mathbb{1}^{\mathrm{b}}$ and Ewen Harrison ${ }^{2} \mathbb{1 0}^{\mathrm{c}}$ \\ ${ }^{1}$ Department of Computer Science, University of Copenhagen, Denmark \\ ${ }^{2}$ Usher Institute, University of Edinburgh, U.K.
}

Keywords: Bayesian Network, Data Mining, Patient Flows, Process Mining, Surgery, Surgical Workflow.

\begin{abstract}
Surgery is a highly critical and costly procedure, and there is an imperative need to improve the efficiency in surgical wards. Analyzing surgical patient flow and predicting cycle times of different peri-operative phases can help improve the scheduling and management of surgeries. In this paper, we propose a novel approach to mining temporal patterns of surgical patient flow with the use of Bayesian belief networks. We present and compare three classes of probabilistic models and we evaluate them with respect to predicting cycle times of individual phases of patient flow. The results of this study support previous work that surgical times are $\log$-normally distributed. We also show that the inclusion of a clustering pre-processing step improves the performance of our models considerably.
\end{abstract}

\section{INTRODUCTION}

Surgery is a cornerstone of the healthcare system, and critical in terms of time and resources. Ensuring efficiency, timeliness and safety are crucial for providing high quality service while controlling costs (Lalys and Jannin, 2014), (Denton, 2007). While many processes surrounding surgery are well structured, the dynamic nature of patient arrivals combined with the complexity of coordinating large numbers of specialized staff and facilities, means that delays and misalignments can have cascading effects leading to last-minute cancellations. This leads not only to an under-utilization of expensive resources, but causes stress and upheaval for patients.

The well-defined, yet dynamic; and high-cost, high-impact nature of surgical patient flows, suggests it is an area amenable to improvements via data oriented process modeling. Advances in forecasting long and short term dynamics of the surgical ward can help inform intelligent surgery sequencing, staff scheduling and workflow management systems.

This paper presents a preliminary investigation into methods for modeling patient flows in surgical wards, with outset in a data set following patients from admission to discharge at the Royal In-

\footnotetext{
a (iD https://orcid.org/0000-0001-7998-7167

b (iD) https://orcid.org/0000-0003-3698-8535

c (iD https://orcid.org/0000-0002-5018-3066
}

firmary of Edinburgh. We focus our present investigation on temporal aspects of individual patient flows, which are key to improving efficiency. Results from this study can then inform the investigation of other aspects of patient flows such as positioning, as well as high-level dynamics between multiple patient flows competing for shared resources at the level of ward/hospital.

After an exploratory investigation of the data, we present and compare three probabilistic models describing cycle times of individual phases in patient flows prior to, during and following surgery. We evaluate these w.r.t. to predicting cycle times of individual phases of patient flows, from the time patients are sent for, through anesthesia and surgery, and until they leave recovery.

Specifically, we employ a type of probabilistic model called a Bayesian network. Aside from their capacity to easily incorporate domain knowledge, Bayesian networks have the advantage that they can be queried in complex ways even with incomplete evidence, which is invaluable in the uncertain hospital environment. Crucially, we show that by incorporating a pre-processing step based on simple clustering of flows w.r.t. cycle times, we can improve the performance of our models noticeably.

The structure of the sequel is as follows. In Section 2 we review existing literature. Our subsequent analysis of the data follows the classic data analytics workflow of Describe $\rightarrow$ Diagnose $\rightarrow$ Predict. In 
Section 3 we introduce the domain, the data set, and the data cleaning process. In Section 4, we present a descriptive analysis of the data set using process mining tools and standard statistical tools to identify informative features of the data. This informs the process of building predictive models which we describe and evaluate in Section 5. In Section 6 we discuss our results and in Section 7 we conclude.

\section{RELATED WORK}

Improving efficiency in surgical wards, specifically improving utilization of operating rooms, has received growing interested nationally and internationally for a number of years now (Lalys and Jannin, 2014). The National Theatres Project in Scotland states as its objective, "appropriately increasing patient throughput, thereby using resources more productively and efficiently"(Scotland, 2006). The metrics for improvement include: reducing unutilized (operating room) hours; reducing over/under-runs, late-starts, cancellations and delayed discharges; and avoiding unnecessary out-of-hours and nighttime procedures. Many of these objectives are strongly related to appropriate scheduling, and would thereby benefit from more accurate, data-informed, models of patient flows.

A significant amount of research exists in modeling processes in the surgical domain. The modeling scope of much existing work tends to fall on two ends of a spectrum in terms of granularity: the level of surgical procedures at one end and broader care flows beyond the surgical ward at the other.

In (Lalys and Jannin, 2014), 46 publications on surgical process modeling are categorized into a taxonomy ranging from the level of the surgical procedure at the lowest level of granularity, to lowlevel physical movements at the highest. At the latter level, which is typically concerned with robotassisted surgery or training and assessment of surgeons, we see research on phase detection (Stauder, 2014) and detailed models of individual tool usage patterns based on sensor data (Ahmadi, 2009). Individual hand motions from video data are automatically identified in (Lin, 2006) and (Haro, 2012). A number of models based on sensor data collected during Cholecystectomies (a highly standardized procedure), were developed in (Blum, 2008), (Bouarfa and Dankelman, 2012), (Bouarfa, 2011), and (Neumuth, 2011). All of these studies have the surgical procedure at the highest level of abstraction. Our present investigation lies above this level of granularity, with only the procedure name and some other basic details being present in the data.

Above the level of individual procedures, we see work such as (Stahl, 2006) which describes the workflow within an operating room, including anesthesia, surgery, and early recovery. Other studies also address the process surrounding surgery, from admission to recovery (Funkner, 2017), which matches the scope of our data set. Taking a view beyond the operating room is important, since activities downstream from the actual surgical procedure can interrupt patient flows as shown in the case of ICU bottlenecks in (Akkerman and Knip, 2004). Some studies have also incorporated diagnosis and follow-up after surgery such as (Mans, 2012) and (Huang, 2013).

Bayesian networks were used to model several aspects of stays in an emergency department in (Acid, 2004). While overall stay duration was one attribute included in the model, the scope was at higher level of abstraction, and not focused specifically on surgical patient flows. Furthermore, the main focus was the comparison of structure learning algorithms.

Some work has looked specifically at modeling variance in surgery durations (Strum, 2000) and incorporating this into sequencing and scheduling strategies (Denton, 2007). In (Kayis, 2012), regression modeling is employed to predict surgery duration based on clinical, operational and temporal data. Stochastic balancing of bed capacity based on fluctuating demand patterns was explored in (Cochran and Bharti, 2006) and length of stay patterns in (Akkerman and Knip, 2004). Resource allocation and patient admission was addressed in (Hulshof, 2013).

In summary, the scope of patient flows ranging from admission, through surgery to recovery, is one which has been less thoroughly addressed: most work is positioned at a lower or higher level of abstraction. In regards to the distribution of surgery times, our work has the corollary contribution of confirming previous findings. In terms of the more nuanced conditional models we present of cycle times, specifically the integration of patient clusters to Bayesian networks, we believe our approach to be novel.

\section{DOMAIN \& DATA PREPARATION}

The Royal Infirmary of Edinburgh is the largest in Scotland, housing 900 beds and with its 24-hour accident and emergency department, providing a full range of acute medical and surgical services. The hospital IT system is integrated with the Operating Room Scheduling Office System (ORSOS), a surgery management and scheduling system. 
The data set analyzed stems from the ORSOS system and involves records ranging from 2010 until 2018 inclusive. Over 1700 types of procedures are recorded in the data set with about half of cases classified as emergency cases. It is oriented around individual surgical procedures, such that any time a patient receives surgical treatment, a new entry is created and each such entry has a unique case ID. This means that the same patient may have multiple unique case IDs, potentially for the same hospital stay. Unique patient IDs, as well as electronic health record identifiers, make it possible to follow patients' overall treatment flows, though this was out of scope of this investigation.

Data regarding patient flows are entered manually by surgical support personnel, with the system requiring the entry of timestamps for each event in the patient flow. Figure 1 illustrates the proscribed sequence of events, and also shows the authors' aggregation of activities into logical phases (pre-op, anesthesia, surgery, recovery). The system enforces a simple linear ordering of events, though it can be overridden. If users attempt to enter timestamps out of sequence, a warning is given, but can be entered upon confirmation. Summaries of cases with anomalous entries are later sent in batches to staff for review.

Aside from the 11 timestamp attributes, the data schema contains 34 other attributes, though some are empty for many cases, such as "reason for delay". Information regarding the procedure performed is included in two different coding schemes, one providing more detail such as location on body. Other case attributes such as the case type (emergency/scheduled), its urgency classification ${ }^{1}$, the ASA patient status rating ${ }^{2}$, and whether the patient is registered as a day-case or inpatient. Staffing details include names of the main and supervising surgeon and anesthetist as well as the consultant assigned to the case. The source of admission (emergency room, etc.), the operating room number, as well as intended and actual destination following surgery (ICU, etc.) are also included. Further details include the diabetic status of the patient, types of anesthetics administered, whether antibiotics were administered, and whether pre-session briefings and surgical pauses were held.

Cleaning and Preparation. The data set contains a number of anomalous entries, comprising roughly $10 \%$ of the 38,728 entries. These entries were re-

\footnotetext{
${ }^{1}$ NCEPOD Classification of Intervention (NCEPOD, 2019).

${ }^{2}$ American Society of Anesthesiologists physical status classification system. (Dripps, 1963)
}

Table 1: Anomalous cases removed prior to analysis.

\begin{tabular}{lrr} 
AnOMALY & COUNT & \% OF TOTAL \\
\hline Duplicate entries & 58 & 0.15 \\
Missing values & 31 & 0.08 \\
Dates out-of-range & 475 & 1.23 \\
Zero timestamps & 3089 & 7.98 \\
Bad ordering & 443 & 1.44 \\
\hline Total & 4096 & 10.58
\end{tabular}

moved prior to further analysis. Table 1 provides an overview.

Duplicate entries may have been due to an attempt to correct a data entry error. The column anaesthetic_start_time was the only timestamp column to contain $\langle\mathrm{NA}\rangle$ values. A larger number of cases have clearly anomalous values in the case_date column, e.g. dates much too far in the past (1800) or future (3206).

Process mining techniques helped quickly reveal that despite the de-jure linear ordering of activities, many anomalous, and decidedly implausible, event orderings exist in the data. Figure 4 shows the result of running the SIMPLE version of the Alpha miner(Van der Aalst, 2004) from the pm4py package (Berti, 2019) on the top 20 sequence variants. The Alpha miner takes as input an event log and outputs a Petri net (specifically a workflow net): a type of process model. Running the Alpha miner on the entire $\log$ results in a flower model ${ }^{3}$. A further a analysis of the directly-follows graph indicated that nearly all possible pairwise event orderings occurred at least once in the data.

One of the aspects of the Petri net in Figure 4 that stands out is that it permits incision_start_time to occur before anaesthetic_start_time. While this was to an extent the result of anomalous timestamps in the data, upon further inquiry with surgical staff, we learned that it is indeed legal for these activities to be recorded with the same timestamp in cases where the surgeon administers a local anesthetic.

Timestamps in the data set are rounded to the minute, and that for many cases, two or more events are recorded with the same timestamp. For example, enter_theatre_time and incision_start_time are sometimes identical, and in fact leave_theatre_time and are enter_recovery_time identical for all cases. This needed to be addressed prior to applying process mining techniques, since they assume sequential orderings in event traces. For this, the de-jure model was used as a tiebreaker in cases of simultaneity.

\footnotetext{
${ }^{3} \mathrm{~A}$ flower model is a process model which permits any event to be executed at any stage of the process.
} 


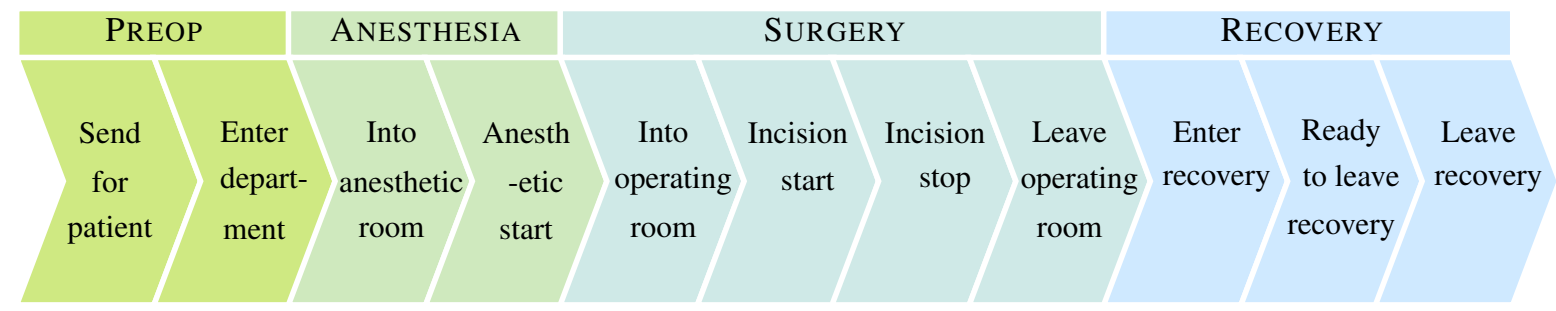

Figure 1: The patient flow proscribed by the ORSOS system. Activities are linearly ordered, but can occur "simultaneously". That is, some activities (such as Leave Operating Room) can have the same timestamp as the "succeeding" activity (Enter Recovery), but should not occur after it.

Many of the implausible cases had zero timestamps associated with the out-of-order events (timestamps of the the form YY-MM-DDT00:00:00). We suspect that these entries may be the result of users specifying only a date without a timestamp. A further 443 cases had anomalous event orderings, likely due to an incorrect entry such as failing to increment the date when a patient flow stretched from one day to the next. All cases with invalid orderings were removed prior to subsequent analysis of cycle time patterns.

Plotting event occurrences on a "dotted chart" (see Figure 2) also reveals several outlying events (occurring months or years from the rest of the flow). The dotted chart simply plots the events from an event by the case id along the $y$-axis and by time along the $x$ axis, such that events associated with the same case fall along a horizontal line.

In the remainder of the analysis, we have removed data points with cycle times in the $99^{\text {th }}$ percentile of values, having observed the presence of events occurring months, even years apart, which for a single surgical case are almost certainly due to data entry mistakes. The chart also makes immediately obvious that a gap exists in the data, and gives an indication of the development in the throughput of cases over time, which remains nearly constant, perhaps increasing slightly.

The number of anomalous cases discovered despite the ORSOS system's compliance measures, demonstrate the importance of data quality measures, especially if such data are to form the basis of prescriptive models and policies. While many of these anomalies would in principle be discoverable by manually querying the data, the use of process mining techniques helped reveal these anomalies quickly and intuitively, serving as a springboard for more detailed analysis.

\section{ANALYSIS}

In this section, we describe the empirical distributions of individual and aggregate cycle times, and compare how well various parametric distributions fit the data. Then, we identify the most informative features of the data set, which will be used as astarting point for model building in 5 .

Marginal Cycle Time Distributions. Fitting an appropriate distribution to data can be a powerful approach to building a predictive model, despite its simplicity. These models consider only the marginal distribution, i.e. they consider outcome across all cases, without conditioning the distribution on case-specific attributes. Table 2 displays the results of fitting seven different distributions to the cycle times of both the original "low-level" events, as well as the aggregated process phases.

As indicated by goodness-of-fit statistics, aggregating individual event cycle times results in more well-formed distributions, with the one slight exception of the recovery stage. While information is clearly lost by reducing 9 cycle times to 4 , this is justified by the fact that any implications of cycle times on resource utilization is captured by the aggregations. For example, an operating theatre, will have the status of being occupied and unavailable for other patients during each of the events Into theatre, Incision start, Incision stop, and until Leave theatre commences. This effect on resource availability is equivalently captured in the aggregations of these events into one Surgery event and its corresponding cycle time.

Previous research has indicated that surgical cycle times are log-normally distributed (Strum, 2000). Our observations are consistent with this, but it should be noted that the Kolmogorov-Smirnov goodness-of-fit does not achieve statistical significance.

Mutual Information. To get an overview of correlation between attributes, the mutual information be- 


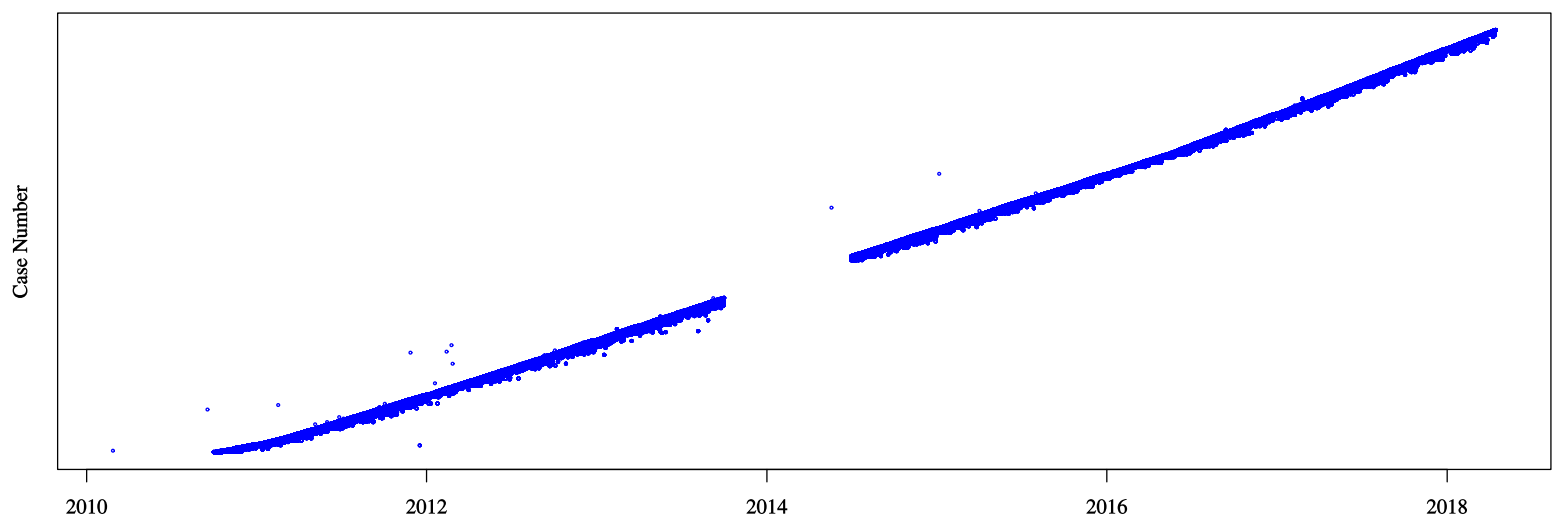

Figure 2: A "dotted chart" helps give a quick overview of the event log. Each data point represents the occurrence of an event, plotted by time on the $x$-axis and by a numerical case identifier on the $y$-axis which are incremented by time. At this level of granularity we cannot see the dynamics of individual process instances, but aspects such as arrival rate, outliers and missing values become clear. In our data set, there is a prominent gap from October 2013 to July 2014, something one would otherwise need to actively investigate, but is immediately noticeable here . Furthermore, several events lie weeks or years from the rest of the events in a case, suggesting anomalous values.

tween attributes was computed, a selection of which are visualized as heatmap in Figure 5. As a nonparametric correlation metric, mutual information is more suitable for our data than parametric estimators such as $\chi^{2}$ since we cannot confidently assume normality for all attributes. Intuitively, mutual information measures the expected decrease in uncertainty regarding the outcome of $y$ upon learning the outcome of $x$. Specifically, it measures the reduction in entropy of the resulting conditional probability distribution.

For continuous values, namely cycle times, it was necessary to discretize the data. This was done such that each of 7 bins contained was of equal widths such that the distribution of cases amongst bins roughly approximates their original distribution. Using this approach, correlations between attributes other than cycle times were the strongest, while attributes influencing cycle times were more weakly correlated, though still observable. Especially intended destination and source of admission standout as informative w.r.t. cycle times. Mutual information can tend to hide important nuances since it reflects the expected value of the pointwise mutual information for individual values of a variable. Lead us to do a more detailed exploration of how different attributes influence cycle times specifically.

Conditional Cycle Time Distributions. By exploring the conditional distributions of cycle times for the individual values attributes, we were able to get a better idea of what influences cycle times. By visualizing conditional distributions on the same plot, one gets a quick impression of the whether an attribute is informative in this respect, or not. Albeit somewhat of a time-consuming, brute-force approach, exploring the data in this way is quite informative. This was an important factor for us in choosing which variables to include in the models we present in Section 5. See Figure 6 for examples of some of the most informative attributes.

Principle Components Analysis. Based on the intuition that cases likely fall into some sort of grouping w.r.t cycle times, we investigated the presence of clusters in the data. For example, cases with a long anesthetic cycle time may also tend to have a long surgery or recovery time - this likely being related to the procedure performed or the patient's condition.

A visual exploration of the raw, as well as $\log$ transformed, data gives the impression that no clear groupings exist. One method for revealing separable clusters in data that are not clearly separable in the original data is via transformation techniques such as Principle Components Analysis (PCA). PCA projects the original data onto a linear subspace which maximizes the resulting variability of the data along the resulting bases, or principle components (Bishop, 2006). It is perhaps most commonly used as a method for dimensionality reduction, by redefining the data on a subset of the principal components which capture most of the variance in the data.

Applying PCA to the log-transformed data reveals that the data does in fact fall into distinct clusters. This can be seen in Figure 3 which shows the data w.r.t to top 3 (of 4) principle components. 
Table 2: Best fits for marginal distributions of cycle times. Goodness-of-fit statistic used is the Kolmogorov-Smirnov criterion.

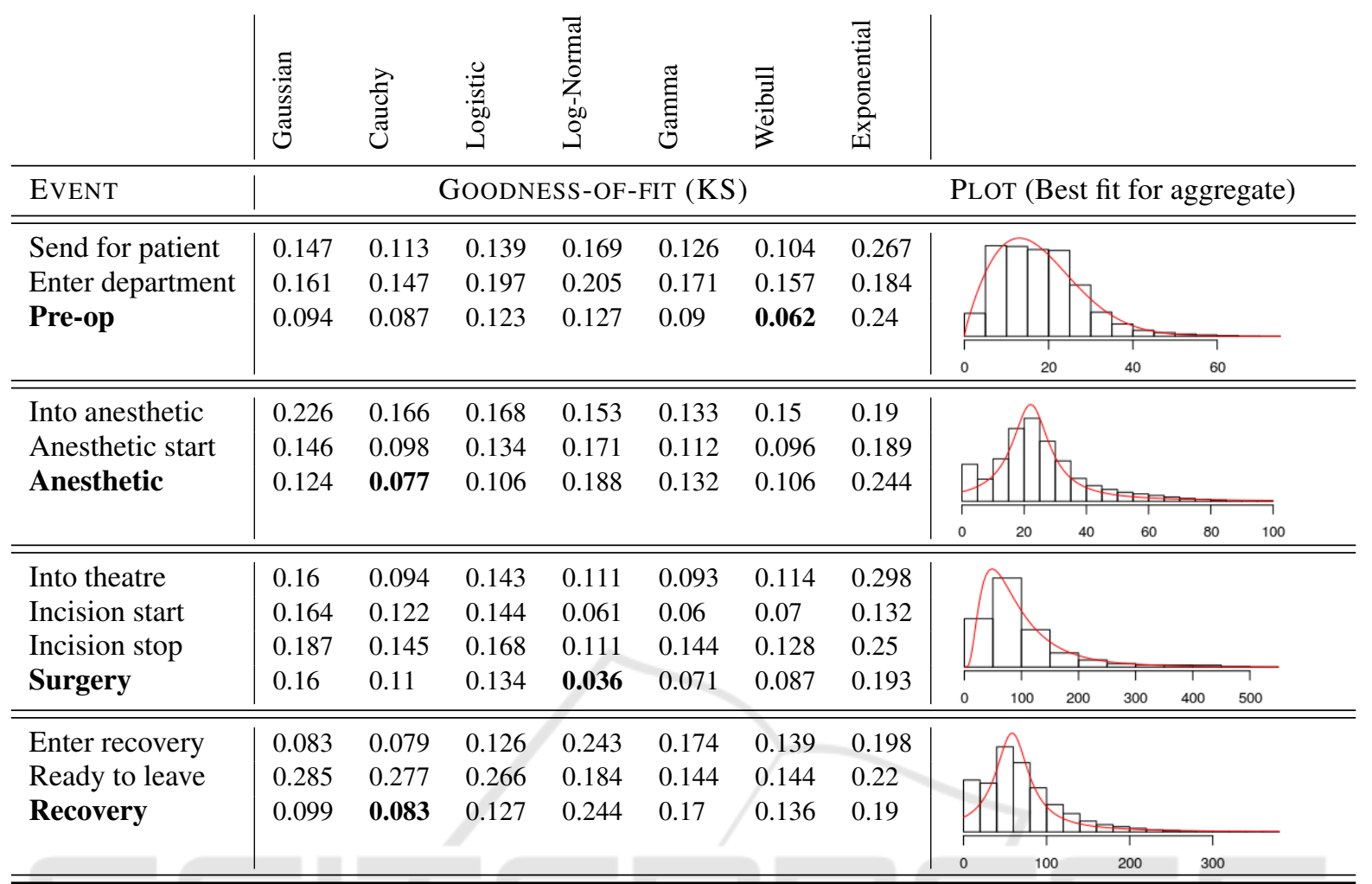

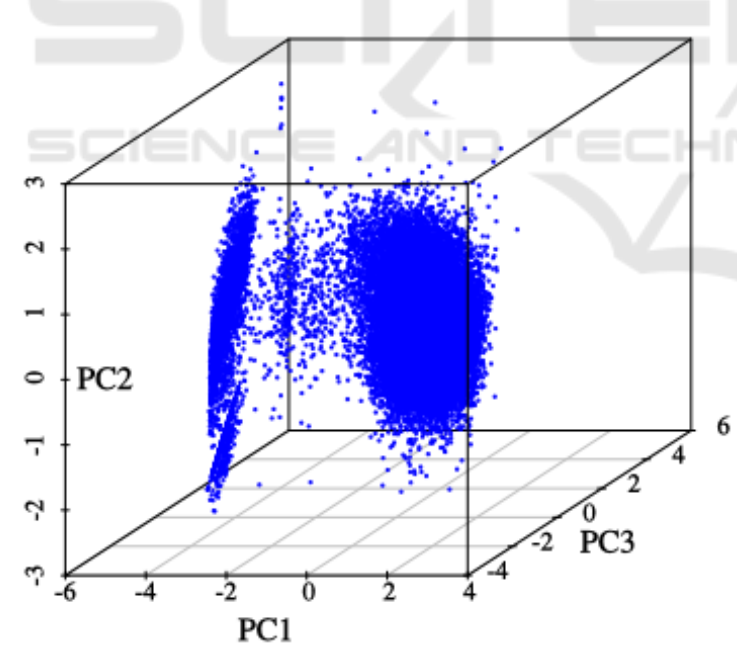

Figure 3: Top 3 principle components for (log-transformed) aggregate cycle times.

\section{PREDICTION}

In order to facilitate improved resource utilization through more accurate scheduling and dynamic resource allocation, we suggest using Bayesian belief networks (Koller and Friedman, 2009). The reason for this choice of model lies in its flexibility. Not limited to one target feature, Bayesian networks can be queried on any attribute, using whatever evidence is currently available. A scheduler can pose queries concerning, for example, the probability of a surgery taking more than $x$ minutes given the case type and condition of patient, or the likely destination of the patient given other evidence.

A Bayesian belief network is a directed acyclic graph with an associated parametrization and represents a joint probability distribution and its conditional independence relations between variables, represented as nodes. Both of these aspects, the graph structure and its parametrization need to be either hand modeled, learned automatically, or a combination of the two. We restricted this investigation to automatically learned models. This can sometimes lead to what may seem counterintuitive models, but it should be kept in mind that an edge between two nodes does not necessarily indicate a causal relationship between source and target.

We present a comparison of 3 classes of models, the latter 2 hybrid discrete/(log)-Gaussian models:

Marginal Model: an unconnected graph, equivalent to the distributions in Table 2.

10 Variable Model: 4 aggregate cycle times, ASA, CaseType, Intended Destination, Management In- 


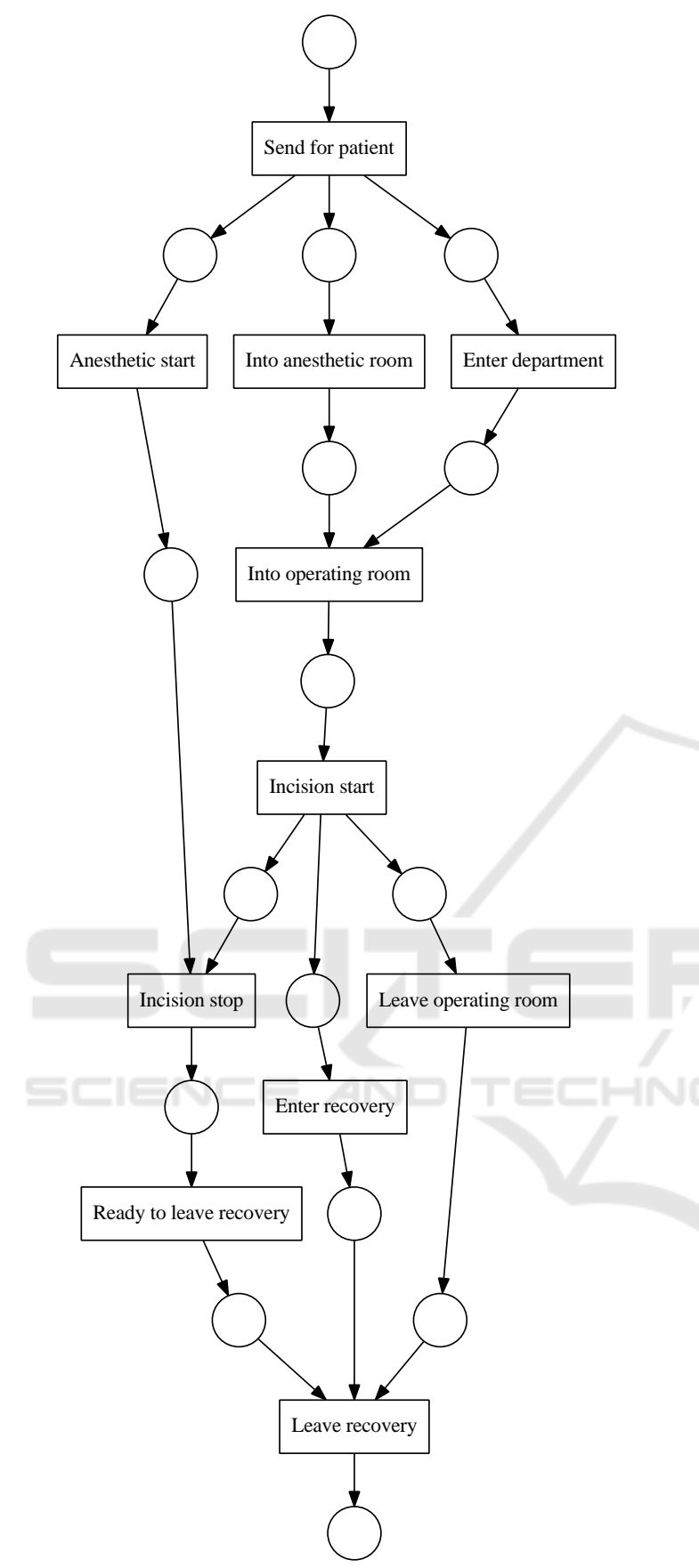

Figure 4: A Petri net generated by the Alpha miner on the top 20 trace variants observed in the event log. This model clearly allows implausible behavior, such as Incision start preceding Anesthetic start.

tent, NCEPOD Category, Source of Admission.

22 Variable Model: 4 aggregate cycle times, ASA, CaseType, Intended Destination, Management Intent, NCEPOD Category, Source of Admission, Diabetic, Operating Room, 10 Anaesthetic Type variables.

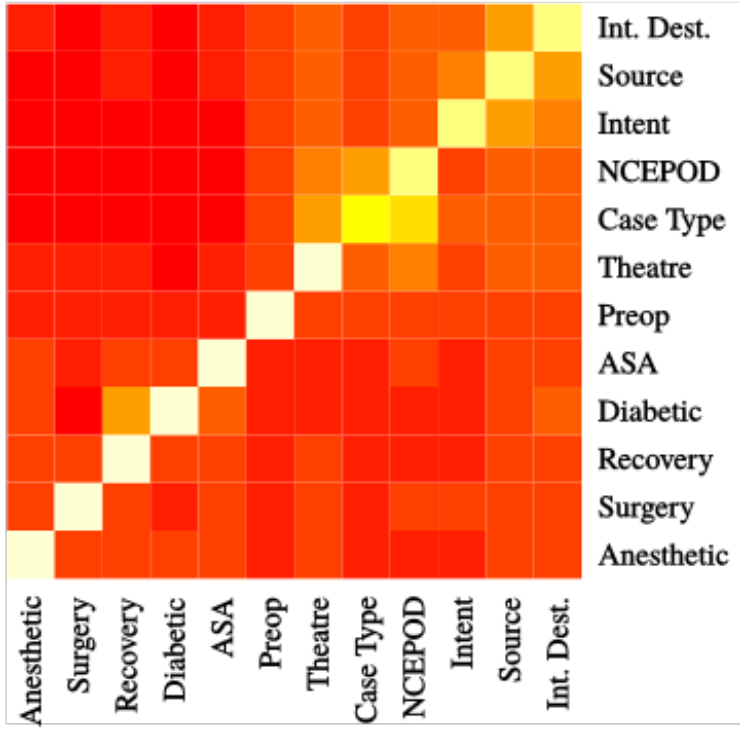

Figure 5: Heatmap of the mutual information between attributes. The "bright" spots indicate that learning the outcome of the corresponding variable on the $x$-axis decreases the uncertainty about the outcome of the corresponding variable on the $y$-axis. Destination denotes the intended destination following the procedure, $N C E P O D$ denotes the urgency classification, and Intent denotes whether the case is a day-case or inpatient case.

Feature Selection. The choice of variables was based on analysis in Section 4, as well as the cardinality of variables. Variables with very large cardinality often fail to improve results due to sparse representation in the data. One solution to allow the incorporation of these is to perform dimensionality reduction on these variables prior to training the network. This is left for future work. For the largest model, we started by including all features, removing those which had no effect on performance.

Clustering. In order to explicitly incorporate the clusters observed in Section 4, we performed simple $k$-means clustering on the PCA transformed data and added a Cluster attribute to each case. This new attribute was then included as a node in some variants of the Bayesian networks. Specifically, we added 4 variants of both the 10- and 22-variable model using different numbers of clusters: $5,10,15,20$. For comparison a model without clusters added is evaluated as well. These values were chosen to illustrate the improvement in model performance upon adding more clusters and the eventual appearance of an elbow of diminishing improvement usually around 15 to 20 clusters. We experimented with values ranging between 2 and 40 . 

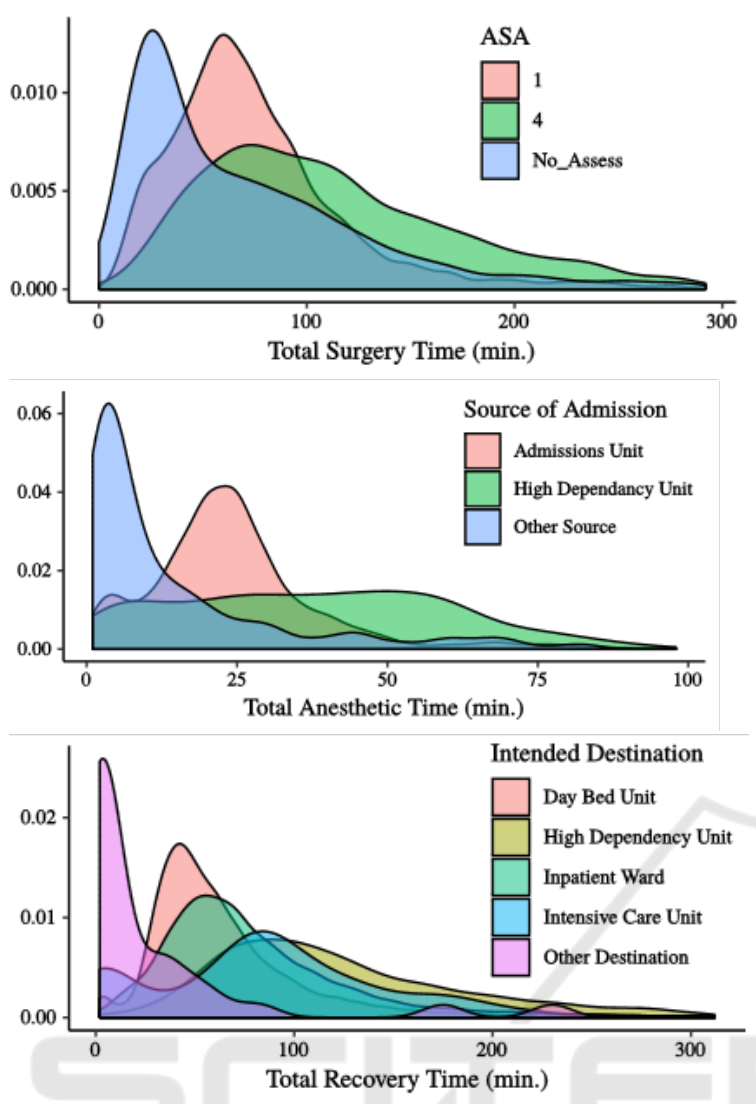

Figure 6: Examples of conditional cycle time distributions. Top: conditioned on ASA status. Middle: Source of Admission. Bottom: Intended Destination.

We found that performing clustering on the PCA transformed data gave slightly better results than clustering on the original data. Note that we used all 4 principal components, hence the data was only transformed and not reduced in dimensionality. It turns out that PCA and $k$-means are in fact closely linked: in (Ding and He, 2004) it is shown that PCA effectively performs clustering w.r.t. the k-means objective function. While performing PCA prior to $k$-means is a widespread practice, it should be noted that it does not always lead to improved results (Yeung and Ruzzo, 2001). We did observe a small improvement over performing $k$-means on the untransformed data.

Learning Algorithms. Structure learning was performed using score-based methods, specifically Hill Climbing and TABU search, using Akaike Information Criterion (AIC), Bayesian Information Criteron (BIC) scores. We were unable to obtain models using log-likelihood scoring within a reasonable time. An example of the graph structure of the learned Bayesian network is shown in Figure 8. Parameter learning was performed using the standard maximum likelihood estimation, partly due to the unavailability of Bayesian estimation techniques for hybrid models in the chosen inference library.

Smoothing \& Priors. Simple smoothing was applied to avoid zero probabilities for outcomes not observed in the training data. This was done by simply adding $0.01 \%$ to all probabilities and subsequently renormalizing. For continuous nodes with discrete parents, the marginal distribution was assigned in case a combination of the parents' values was observed in the training data.

Evaluation. Evaluation of Bayesian networks is often based on quantifying how closely the probability distribution represented by the network matches the empirical distribution (data). Typical metrics include log likelihood, Akaike information criterion, Bayesian information criterion, and Kullback-Liebler divergence.

One can also consider a specific target variable for prediction and measure the error rate. Since we are specifically interested in predicting cycle times, we report results of the mean absolute error of predictions for these 4 target variables. We chose to report this metric rather than the more standard (root) mean squared error (RMSE), since it gives a more immediate sense of how far predictions were from actual cycle times in terms of the original time units (minutes). Results for RMSE follow very nearly the same pattern between models. Results are reported for 5 runs of 10-fold cross-validation, see Figure 7.

A crucial point regarding our approach to cross validation concerns which attributes were considered observed at each phase of the process. When predicting a given cycle time, clearly any future cycle times will not have been observed and should not be included as inputs to the model. So when predicting the Preop cycle time, Anesthesia, Surgery, and Recovery should not be including as inputs. However, once the patient has reached the Recovery phase, the preceding cycle times are at least theoretically known and can be used as inputs. This was our approach, motivated by the assumption that even if patient flow monitoring systems do not presently integrate such real-time information they will likely do so in the near future.

\section{DISCUSSION}

Our preliminary analysis of the ORSOS data set has demonstrated three points. First, the importance of data quality assurance, cleaning and the usefulness 

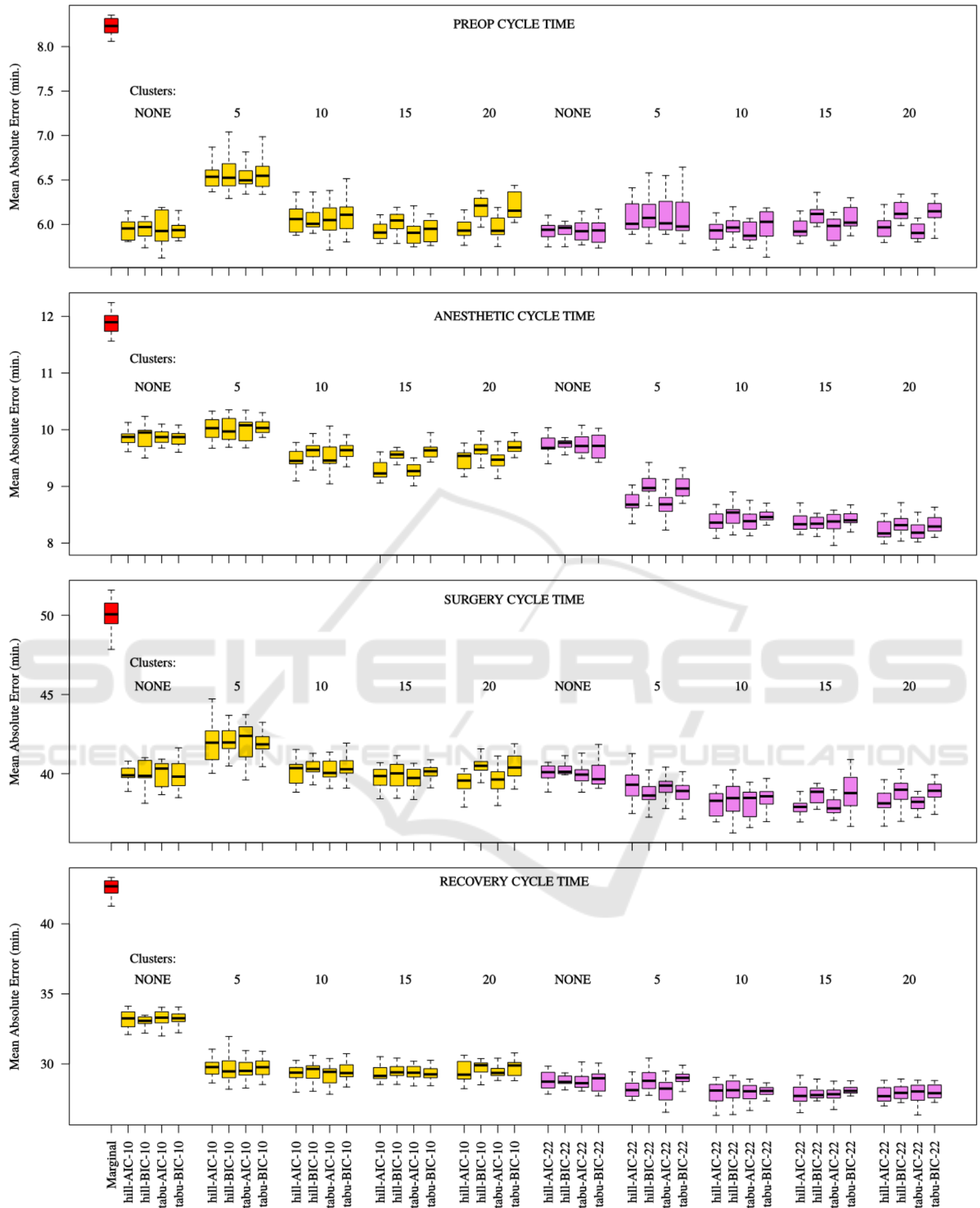

Figure 7: 5 runs of 10-fold cross-validation based on mean absolute error on the 4 cycle time target variables: preop, anesthetic, surgery, and recovery. Red: Marginal baseline model. Gold: 10-variable model. Violet: 22-variable model. Within each boxplot grouping are results for models learned with the Hill Climbing and TABU structure learning algorithms using Akaike Information Criterion (AIC) and Bayesian Information Criterion (BIC) respectively. 


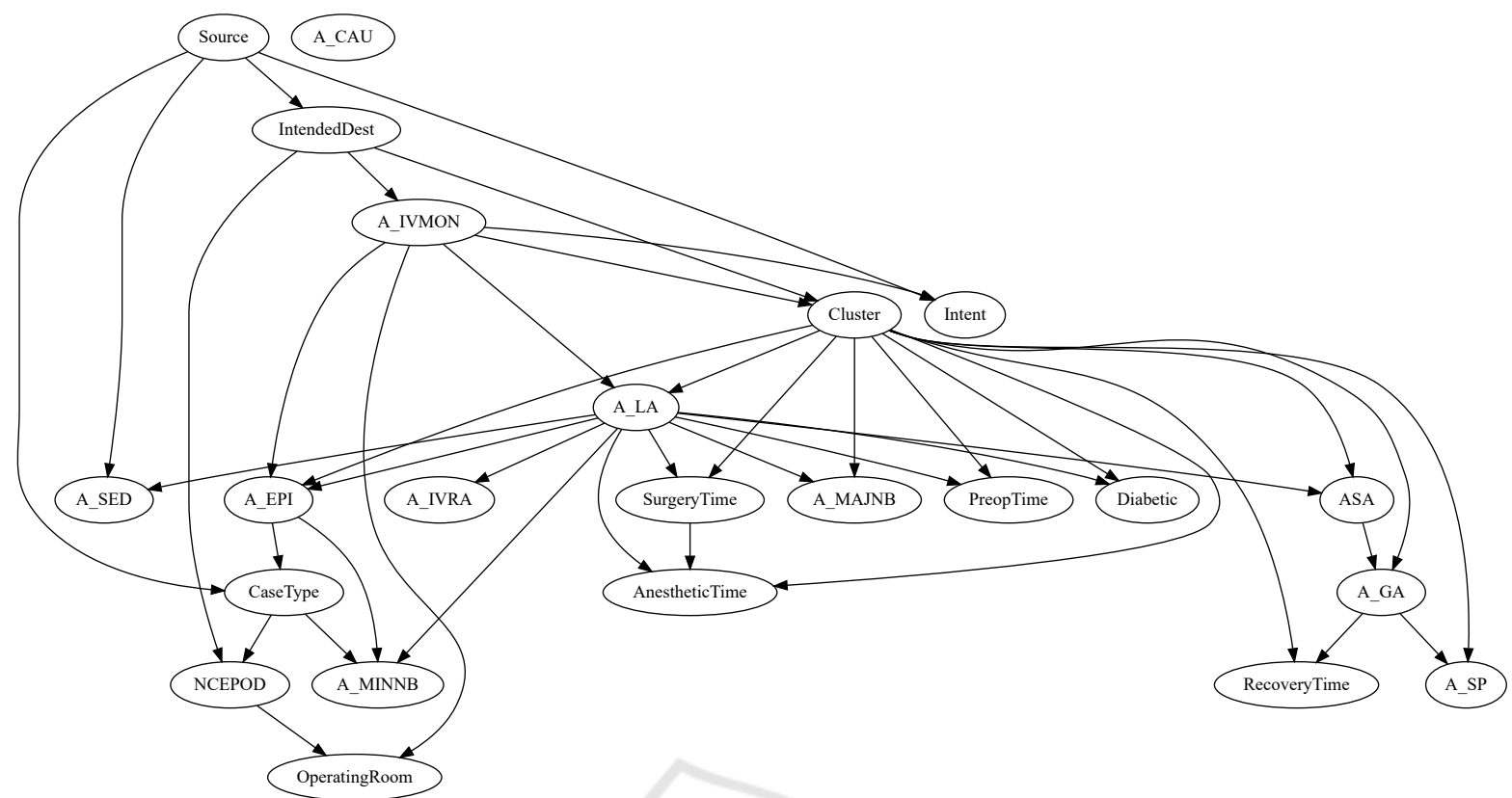

Figure 8: The overall best performing model. The 22 variable model using TABU with BIC scoring and 20 clusters. Node labels prefixed by Ane. denote anaesthetic types. NCEPOD indicates urgency classification, ASA patient condition and Intent indicates day-case/inpatient.

of process mining techniques in this respect. Second, that a reasonably accurate predictive model of event cycle times in the form of a simple Bayesian belief network can be built which significantly outperforms simple marginal distribution fitting. Third, that by clustering the target variables and including these cluster labels as attribute in the model and training data improves accuracy yet further. Furthermore, we describe how mutual information, tools for exploring conditional probability distributions and principle components analysis can not only give insight into the data, but also guide model building

The choice of Bayesian networks was motivated by their flexibility and interpretability. The fact that they can be queried in such a versatile manner, based on whatever data is available at the time, suggests they would be a strong component of a predictive model in a decision support and scheduling systems in surgery. This allows for queries of the form, "what is the probability that case $c$ will be in surgery for more than $m$ minutes given it has the following attributes, and took $n$ minutes to complete anesthesia?". Specifically, these could form the basis for probabilistic scheduling systems.

\section{CONCLUSION \& FUTURE WORK}

We have demonstrated the utility of combining several data analysis tools, including from process mining and machine learning, to begin building a useful model of a very complex set of processes in a surgical ward. This approach would be also applicable in other areas of the healthcare system in which under-utilization of expensive resources calls for precise scheduling to avoid down-time.

In terms of the full data analytics workflow, often summarized by Describe $\rightarrow$ Diagnose $\rightarrow$ Predict $\rightarrow$ Prescribe, we have only just begun the Predict phase. The incorporation of more aspects of the data set is a clear next step - the huge cardinality of some attributes, such as procedures and staff, should be addressed by incorporating domain knowledge and/or dimensionality reduction. Considering the notable improvements in precision we achieved with a relatively limited data set, it is likely that incorporating patient flow attributes across domains would lead to yet more precise models.

A thorough comparison with learning algorithms other than simple distribution fitting to confirm the suitability of Bayesian networks to this application is also important. Finally, while we have focused on individual patient flows here, more comprehensive 
models which take into account ward level dynamics such as patient arrival rates, resource constraints, and resulting inter-patient dynamics are a natural extension. In such a system-wide model, the work presented here would serve as a component to more accurately model local event timings and subsequent downstream arrival rates.

\section{REFERENCES}

Acid, S., e. a. (2004). A comparison of learning algorithms for bayesian networks: a case study based on data from an emergency medical service. Artificial intelligence in medicine, 30(3):215-232.

Ahmadi, S.A., e. a. (2009). Motif discovery in or sensor data with application to surgical workflow analysis and activity detection. In M2CAI workshop, MICCAI, London. Citeseer.

Akkerman, R. and Knip, M. (2004). Reallocation of beds to reduce waiting time for cardiac surgery. Health care management science, 7(2):119-126.

Berti, A., e. a. (2019). Process Mining for Python (PM4Py): Bridging the Gap Between Process-and Data Science. In ICPM Demo Track (CEUR 2374).

Bishop, C. M. (2006). Pattern recognition and machine learning. Springer Science+ Business Media.

Blum, T., e. a. (2008). Workflow mining for visualization and analysis of surgeries. Int. journal of computer assisted radiology and surgery, 3(5):379-386.

Bouarfa, L., e. a. (2011). Discovery of high-level tasks in the operating room. Journal of biomedical informatics, 44(3):455-462.

Bouarfa, L. and Dankelman, J. (2012). Workflow mining and outlier detection from clinical activity logs. Journal of biomedical informatics, 45(6):1185-1190.

Cochran, J. K. and Bharti, A. (2006). Stochastic bed balancing of an obstetrics hospital. Health care management science, 9(1):31-45.

Denton, B., e. a. (2007). Optimization of surgery sequencing and scheduling decisions under uncertainty. Health care management science, 10(1):13-24.

Ding, C. and He, X. (2004). K-means clustering via principal component analysis. In Proc. of the twenty-first int. conference on Machine learning, page 29. ACM.

Dripps, R. (1963). American society of anesthesiologists. New classification of physical status. Anesthesiology, 24(1):111.

Funkner, A. A., e. a. (2017). Towards evolutionary discovery of typical clinical pathways in electronic health records. Procedia computer science, 119:234-244.

Haro, B.B., e. a. (2012). Surgical gesture classification from video data. In Int. Conf. on Medical Image Computing and Computer-Assisted Intervention, pages 3441. Springer.

Huang, Z., e. a. (2013). Summarizing clinical pathways from event logs. Journal of biomedical informatics, 46(1):111-127.
Hulshof, P. J. H., e. a. (2013). Tactical resource allocation and elective patient admission planning in care processes. Health care management science, 16(2):152166.

Kayis, E., e. a. (2012). Improving prediction of surgery duration using operational and temporal factors. In AMIA Annual Symposium Proc., volume 2012, page 456. American Medical Informatics Association.

Koller, D. and Friedman, N. (2009). Probabilistic graphical models: principles and techniques. MIT press.

Lalys, F. and Jannin, P. (2014). Surgical process modelling: a review. Int. journal of computer assisted radiology and surgery, 9(3):495-511.

Lin, H.C., e. a. (2006). Towards automatic skill evaluation: Detection and segmentation of robot-assisted surgical motions. Computer Aided Surgery, 11(5):220-230.

Mans, R., e. a. (2012). Mining processes in dentistry. In Proc. of the 2nd ACM SIGHIT Int. Health Informatics Symposium, pages 379-388. ACM.

NCEPOD (2019). NCEPOD classification of intervention. https://www.ncepod.org.uk/classification.html. Accessed: 2019-11-22.

Neumuth, T., e. a. (2011). Analysis of surgical intervention populations using generic surgical process models. Int. Journal of Computer Assisted Radiology and Surgery, 6(1):59-71.

Scotland, N. (2006). National theatres project report. https://www.isdscotland.org/Health-Topics/ Quality-Indicators/National-Benchmarking-Project/ National-Theatres-Project/. Accessed: 2019-11-22.

Stahl, J. E., e. a. (2006). Reorganizing patient care and workflow in the operating room: a cost-effectiveness study. Surgery, 139(6):717-728.

Stauder, R., e. a. (2014). Random forests for phase detection in surgical workflow analysis. In Int. Conf. on Information Processing in Computer-Assisted Interventions, pages 148-157. Springer.

Strum, D., e. a. (2000). Modeling the uncertainty of surgical procedure times: comparison of log-normal and normal models. Anesthesiology, 92(4):1160-1167.

Van der Aalst, W. e. a. (2004). Workflow mining: Discovering process models from event logs. IEEE Transactions on Knowledge and Data Engineering, 16(9):1128-1142.

Yeung, K. and Ruzzo, W. L. (2001). An empirical study on principal component analysis for clustering gene expression data. Bioinformatics, 17(9):763-774. 